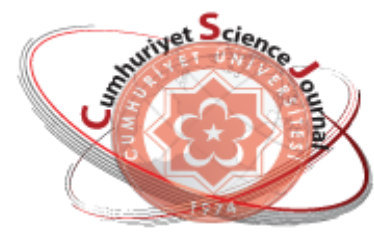

e-ISSN: $2587-246 X$

ISSN: $2587-2680$

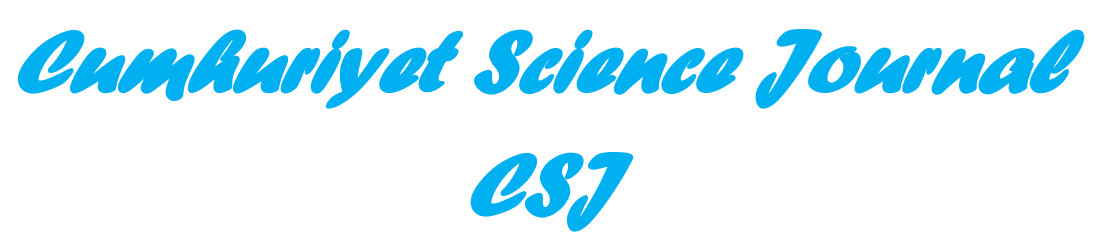

Cumhuriyet Sci. J., Vol.40-3 (2019) 612-623

\title{
Double Actions of Groups
}

Mustafa Habil GÜRSOY

Inönü University, Faculty of Arts Sciences, Department of Mathematics, Malatya-44280, TURKEY

\begin{abstract}
In this work, we present the concept of double action of a group $G$ on a set $X$ as a new concept in literature, which opens up the way for applying rich amount of algebraic topological facts and methods in the algebraic topology. Besides of this, we give some expository examples of this new concept. We also express the definition of a double action via permutations as similar to the definition of an action on a set of groups via permutations. We investigate some characterizations about the double actions. Also we prove an adaptation of Cayley's theorem in the case of the double action.
\end{abstract}

Keywords: Group; action; double action.

\section{Grupların Çift Etkileri}

Özet. Bu çalışmada, literatürde yeni bir kavram olarak bir G grubunun bir X kümesi üzerine çift etkisi kavramını sunuyoruz, ki bu kavram cebirsel topolojide cebirsel topolojik gerçeklerin ve metotların zengin bir uygulama yolunu açacaktır. Bunun yanısıra, bu yeni kavramın bazı açıklayıcı örneklerini veriyoruz. Ayrıca permütasyonlar yardımıyla grupların bir küme üzerine etkisi tanımına benzer olarak permütasyonlar yardımıyla bir çift etkinin tanımını ifade ediyoruz. Çift etkiler hakkında bazı karakterizasyonları inceliyoruz. Ayrıca çift etki durumunda Cayley's teoreminin bir uyarlamasını ispatllyoruz.

Anahtar Kelimeler: Grup; etki; çift etki.

\section{INTRODUCTION}

The concept of action is an important tool in many area of mathematics, especially in algebraic topology. For instance, some categorical equivalences have been shown using the action of groupoid [2, 4]. Besides of the group actions, the actions of other algebraic structures such as algebra, ring, algebroid and groupoid are also studied $[1,5]$.

An action of a group $G$ on a set $X$ is a map $\cdot: G \times X \rightarrow X$ satisfying the conditions $e \cdot x=x$ and $g \cdot(h \cdot x)=$ ( $g h) \cdot x$ for all $g, h \in G$ and $x \in X$, where $e$ is the identity of $G$. On the other hand, we can also define the action by using the concept of permutation, namely, an action of a group $G$ with identity $e$ on a set $X$ is the choice of a permutation $\tau_{g}: X \rightarrow X$ for each $g \in G$ such that $\tau_{e}$ is the identity and, $\tau_{g} \circ \tau_{h}=\tau_{g h}$ for all $g, h \in G$. Furthermore, two basic concepts come to mind when dealing with group action: orbit and stabilizer. If there exists an action of $G$ on $X$, then the set $\operatorname{Orb}_{G} x=\{g \cdot x \mid g \in G\}$ is called the orbit of $x$. The stabilizer of $x$ is the set $G_{x}=\{g \in G \mid g \cdot x=x\}$ which is a subgroup of $G$.

In this work, we deal with double actions of groups on sets. We investigate double situations of the concepts mentioned above. For this aim, we give definition of double action of a group on a set. In more 
detail, we define the double action as follows: Let us suppose that a group $G$ has two actions on a set $X$ on the left as ". " and "० ". Then, for every $g, h \in G$ and $x \in X$, if $g \cdot\left(h^{\circ} x\right)=h^{\circ}(g \cdot x)$ holds, then we say that $G$ acts doubly on $X$ on the left by means of ". "and " $\circ "$. Using the concept of permutation, we can redefine the double action as follows. We say that $G$ acts doubly on $X$, if it is possible to correspond a permutation $\eta_{g}^{h}$ of $X \times X$ to each pair of elements $(g, h) \in G \times G$ such that $\eta_{g}^{h} \circ \eta_{k}^{m}=\eta_{g k}^{h m}$ for every $(g, h),(k, m) \in G \times G$. Also, we present the concepts of double orbit and stabilizer for double actions. Let $G$ be a group doubly acting on a set $X$ by means of ". " and " $\circ$ " and let $x \in X$. Then, the double orbit of $x$ is the set $\operatorname{DOrb}_{G}(x)=\left\{y \in X: g \cdot x=y=h^{\circ} x, g, h \in G\right\}$, and the stabilizer of $x$ is the set $G_{x}=\left\{(g, h) \in G \times G: g \cdot x=h^{\circ} x=x, g, h \in G\right\}$ which is the subgroup of the product group $G \times G$. Furthermore, we present the equivalence of two double actions, namely, we say that double actions of $G$ on sets $X$ and $X^{\prime}$ are equivalent if there is a bijection $\phi: X \rightarrow X^{\prime}$ such that $\phi(g \cdot x)=$ $g(\phi(x))$ and $\phi\left(h^{\circ} x\right)=h^{\circ}(\phi(x))$ for every $g, h \in G$ and $x \in X$. Additionally, we define the concepts of transitive, effective, free and regular double actions. Besides of these, we prove an adaptation of Cayley's theorem in the case of double action of groups.

We consider that the concept of double action as a new concept will bring a new look to problems particularly those in algebraic topology. Moreover, the concept of double action for the above mentioned algebraic structures can be also defined.

\section{PRELIMINARIES}

This section of the paper is devoted to give basic facts related to the actions of groups on sets.

Definition 1. Let $G$ be a group and $X$ a nonempty set. A left action of $G$ on $X$ is a map $\cdot: G \times X \rightarrow$ $X$ written as $(g, x) \mapsto g \cdot x$ satisfying

i) $e \cdot x=x$ for all $x \in X$, where "e" denotes the identity element of the group $G$.

ii) $g \cdot(h \cdot x)=(g h) \cdot x$ for all $g, h \in G$ and $x \in X[2]$.

Given an action of $G$ on $X$, we call $X$ a $G-$ set. We denote the action by $(G, ; X)$. Similarly, a right action of $G$ on $X$ is defined. In this case, the group elements are written on the right instead of the left. Namely, the first condition is written by $x \cdot g=x$, and the second condition is written by $(x \cdot g)^{\cdot h}=x \cdot(g h)$. But, we will use the left action throughout the paper.

Example 1. Let $G$ be a group and $X=G$. Then $G$ acts on itself by left multiplication [2].

Example 2. Let $G$ be a group and $X=G$. Then $G$ acts on itself by conjugation [2].

Example 3. The trivial action of any group $G$ on any set $X$ is defined by $g \cdot x=x$ for all $g \in G$ and all $x \in X[2]$.

Another way to think about an action of a group on a set is via the concept of symmetric group, which this alternative definition will give us a very important theorem related to the group action: Cayley's theorem. For this aim, let us first recall the concept of symmetric group.

Let $X$ be any set. A permutation of $X$ is a function $\tau: X \rightarrow X$ which is invertible (i.e. it is both injective and surjective). The set of permutations of $X$ forms a group under composition of functions, called the symmetric group on $X$. We denote it by $\operatorname{Sym}(X)$. Indeed, since the identity function $I d_{X}$ on $X$ is a bijection 
from $X$ to $X$, we have $\operatorname{Id}_{X} \in \operatorname{Sym}(X)$, and so $\operatorname{Sym}(X) \neq \varnothing$. For any $\tau, \phi \in \operatorname{Sym}(X)$, since the composition $\tau \circ \phi$ is a bijection from $X$ to $X$, we have $\tau \circ \phi \in \operatorname{Sym}(X)$. Also, it is clear that the operation $\circ$ has associative property. For all $\tau \in \operatorname{Sym}(X)$, we have $\tau^{-1} \in \operatorname{Sym}(X)$. It follows $\tau$ 。 $\tau^{-1}=I d_{X}=\tau^{-1} \circ \tau$. Thus, $(\operatorname{Sym}(X), \circ)$ is a group.

It is clear that if we take a group $G$ instead of the set $X$, then we obtain group of the automorphisms of $G$.

Now let us again define the action of a group $G$ on a set $X$ via the concept of permutation.

Definition 2. An action of a group $G$ on a set $X$ is the choice of a permutation $\tau_{g}: X \rightarrow X$ for each $g \in G$ such that the following two conditions hold:

i) $\tau_{e}$ is the identity, namely $\tau_{e}(x)=x$ for each $x \in X$,

ii) for every $g, h \in G, \tau_{g} \circ \tau_{h}=\tau_{g h}[3]$.

If we consider together the Definitions 1. and 2., then we can state the following proposition which is give the relation between the group $G$ and the symmetric group $\operatorname{Sym}(X)$ of the permutations of $X$ via the concept of action of the group $G$ on the set $X$ :

Proposition 1. An action of a group $G$ on a set $X$ is the same as a group homomorphism from $G$ to $\operatorname{Sym}(X)$ [3].

By this proposition, we can easily say that there is no difference between an action of a group $G$ on a set $X$ and a homomorphism $f: G \rightarrow \operatorname{Sym}(X)$.

We can now state Cayley's theorem.

Theorem 1. Every group $G$ is isomorphic to a group of permutations [3].

Definition 3. Let $G$ be a group acting on a set $X$. If $x \in X$, then the set $\operatorname{Orb}_{G} x=\{g \cdot x \mid g \in G\}$ is called the orbit of $x$ or the orbit through $x[2]$.

The set of orbits under the action of $G$ forms a partition of $X$. Because they are the equivalence classes of the equivalence relation given by

$$
y \sim x \Leftrightarrow y=g \cdot x \text { for some } g \in G .
$$

The set of all orbits of $X$ under the action of $G$ is written as $X / G$.

Definition 4. Let $X$ be a $G$ - set. Let $x \in X$. The stabilizer of $x$ is the set $G_{x}=\{g \in G \mid g \cdot x=x\}$ [2].

Proposition 2. The stabilizer $G_{x}$ of $x$ is a subgroup of $G$ [2].

Definition 5. Let $X$ be a $G-$ set. Then

i) the action is transitive if $X \neq \emptyset$ and if for any $x, y \in X$, there exists $g \in G$ such that $g: x=y$.

ii) the action is faithful if for any $g, h \in G$, there exists $x \in X$ such that $g \cdot x \neq h \cdot x$. 
iii) the action is free if for $g, h \in G$, if there exists $x \in X$ such that $g \cdot x=h \cdot x$ implies $g=h$.

iv) the action is regular if it is both free and transitive. Equivalently, we have that for any $x, y \in X$ there exists a unique $g \in G$ such that $g \cdot x=y[2]$.

\section{DOUBLE ACTIONS OF GROUPS}

Definition 6. Let $G$ be a group and $X$ be a nonempty set. Let us suppose that $G$ has two actions on $X$ on the left as ". " and " $\circ "$. For every $g, h \in G$ and $x \in X$, if the interchange law

$$
g \cdot\left(h^{\circ} x\right)=h^{\circ}(g \cdot x)
$$

holds, then we say that $G$ acts doubly on $X$ on the left by means of". " and " ॰". The action of $G$ on $X$ defined by this way is called left double action. Then, the set $X$ is called a left double $G-$ set. We denote the left double action of $G$ on $X$ by $(G, \cdot, \circ, X)$.

Let's state that there is no need in the above definition for a condition similar to condition (i) related to the identity element given in Definition.1. Namely, since we have already had two actions such as "." and " $\circ$ ", the equalities $e \cdot x=x$ and $e^{\circ} x=x$ are satisfied by themselves. And, when we take identity element " $e$ " in the interchange law (*), it is obvious that the interchange law is satisfied.

Example 4. Let us define two different actions of a group $G$ on itself on the left as follows:

$$
\begin{gathered}
\cdot G \times G \rightarrow G \\
(g, x) \mapsto g \cdot x=g x,
\end{gathered}
$$

and

$$
\begin{gathered}
\circ: G \times G \rightarrow G \\
(h, x) \mapsto h^{\circ} x=x h^{-1}
\end{gathered}
$$

Then, the group $G$ acts doubly on itself on the left by means of ". " and " $\circ$ ". Namely, for all $g, h, x \in G$, we have the equality

$$
g \cdot\left(h^{\circ} x\right)=g \cdot\left(x h^{-1}\right)=g\left(x h^{-1}\right)=(g x) h^{-1}=h^{\circ}(g x)=h^{\circ}(g \cdot x) .
$$

That is, the condition (*) in Definition. 6 holds. Consequently, $(G, \cdot, \circ, G)$ is a left double action.

Example 5. Let us consider the following two actions of a group $G$ on itself on the left.

$$
\begin{gathered}
\cdot: G \times G \rightarrow G \\
(g, x) \mapsto g \cdot x=g x g^{-1},
\end{gathered}
$$

and

$$
\begin{gathered}
\circ: G \times G \rightarrow G \\
(h, x) \mapsto h^{\circ} x=x
\end{gathered}
$$


Then, for every $g, h, x \in G$, since

$$
g \cdot\left(h^{\circ} x\right)=g \cdot(x)=g x g^{-1}=h^{\circ}\left(g x g^{-1}\right)=h^{\circ}(g \cdot x)
$$

the group $G$ acts doubly on itself on the left by means of ". " and " ०". Thus, $(G, \cdot, \circ, G)$ is a left double action.

Proposition 3. If a group $G$ is abelian, every left action of $G$ on the set $X$ is a left double action.

Proof. The proof follows easily from that the second condition of the action and the commutativity of the group. Namely, let $G$ be an abelian group and $G$ acts on a nonempty set $X$ on the left. Let us denote the action by $\cdot: G \times X \rightarrow X,(g, x) \mapsto g \cdot x$. Then, for every $g, h \in G$ and $x \in X$, we have

$$
g \cdot(h \cdot x)=(g h) \cdot x=(h g) \cdot x=h \cdot(g \cdot x) .
$$

Thus, the abelian group $G$ acts doubly on $X$ on the left by the action ". ", which completes the proof.

Now, our current aim is to define double actions via permutations just as we have defined group actions via permutations.

If $(G, \cdot, X)$ is an action, then we have a homomorphism

$$
\begin{aligned}
\theta: G & \rightarrow \operatorname{Sym}(X) \\
\qquad \mapsto \theta(g)=\varphi_{g}: X & \rightarrow X \\
& x \mapsto \varphi_{g}(x)=g \cdot x
\end{aligned}
$$

such that $\theta(g h)=\varphi_{g h}=\varphi_{g} \circ \varphi_{h}=\theta(g) \circ \theta(h)$.

Similarly, if $(G, \circ, X)$ is another action, then we have also a homomorphism

$$
\begin{aligned}
\Gamma: G & \rightarrow \operatorname{Sym}(X) \\
h \mapsto \Gamma(h)=\psi^{h}: & X \rightarrow X \\
& x \mapsto \psi^{h}(x)=h^{\circ} x
\end{aligned}
$$

such that $\Gamma(g h)=\psi^{g h}=\psi^{g} \circ \psi^{h}=\Gamma(g) \circ \Gamma(h)$.

After all, let $(G, \cdot, \circ, X)$ be a double action. Then, let us define

$$
\begin{aligned}
\Phi: G \times G \rightarrow \operatorname{Sym}(X \times X) & \\
(g, h) \mapsto \Phi(g, h)=\eta_{g}^{h}: X \times X \rightarrow X \times X & \\
(x, y) \mapsto \eta_{g}^{h}(x, y) & =\left(\varphi_{g}(x), \psi^{h}(y)\right) \\
& =\left(g \cdot x, h^{\circ} y\right) .
\end{aligned}
$$


Unlike the homomorphisms $\theta$ and $\Gamma$ just mentioned above, it is not so difficult to understand that $\Phi$ is defined on $G \times G$ because of its double state.

It is obvious that the $\operatorname{Sym}(X \times X)$ is a group. Namely, since $\eta_{e}^{e}(x, y)=\left(e \cdot x, e^{\circ} y\right)=(x, y)$ for all $(x, y) \in X \times X$, then $\eta_{e}^{e}$ is the identity element of $\operatorname{Sym}(X \times X)$, where $(e, e)$ is the identity of $G \times G$. Also the multiplication of $\operatorname{Sym}(X \times X)$ is defined by

$$
\begin{aligned}
\eta_{g}^{h} \circ \eta_{k}^{m}(x, y) & =\eta_{g}^{h}\left(\eta_{k}^{m}(x, y)\right) \\
& =\eta_{g}^{h}\left(k \cdot x, m^{\circ} y\right) \\
& =\left(g \cdot(k \cdot x), h^{\circ}\left(m^{\circ} y\right)\right) \\
& =\left((g k) \cdot x,(h m)^{\circ} y\right) \\
& =\eta_{g k}^{h m}(x, y)
\end{aligned}
$$

for all $(g, h),(k, m) \in G \times G$ and $(x, y) \in X \times X$.

The inverse of $\eta_{g}^{h}$ is defined by $\eta_{g^{-1}}^{h^{-1}}$ for any $(g, h) \in G \times G$.

Now let us show that $\Phi$ defined above is a homomorphism.

For any $(g, h),(k, m) \in G \times G$ and $(x, y) \in X \times X$,

$$
\begin{aligned}
\Phi((g, h)(k, m))(x, y) & =\Phi(g k, h m)(x, y) \\
& =\eta_{g k}^{h m}(x, y) \\
& =\left((g k) \cdot x,(h m)^{\circ} y\right) \\
& =\left(g \cdot(k \cdot x), h^{\circ}\left(m^{\circ} y\right)\right) \\
& =\eta_{g}^{h}\left(k \cdot x, m^{\circ} y\right) \\
& =\eta_{g}^{h}\left(\eta_{k}^{m}(x, y)\right) \\
& =\Phi(g, h) \Phi(k, m)(x, y) .
\end{aligned}
$$

Hence, $\Phi$ is a homomorphism.

Considering all of these, we can also give a new definition of a double action of $G$ on $X$ as follows, equivalently to the Definition 6:

Definition 7. Let $X$ be a set and $G$ a group. Then, we say that $G$ acts doubly on $X$, if it is possible to correspond a permutation $\eta_{g}^{h}$ of $X \times X$ to each pair of elements $(g, h) \in G \times G$ such that $\eta_{g}^{h} \circ \eta_{k}^{m}=\eta_{g k}^{h m}$ for every $(g, h),(k, m) \in G \times G$. 
In other words, if there exists a homomorphism from $G \times G$ to $\operatorname{Sym}(X \times X)$, then it is called " $G$ acts doubly on X".

After this definition, we state the Cayley's theorem in case of the double action.

Theorem 2. Given a group $G$, the group $G \times G$ is isomorphic to a group of permutations.

Proof. For the proof, just as in the Theorem 1., we are going to utilize the advantage of the idea that the left-multiplication is a permutation in the light of the above explanations for the group $G \times G$.

Define a map

$$
\begin{gathered}
\Phi: G \times G \rightarrow \operatorname{Sym}(G \times G) \\
(g, h) \mapsto \Phi(g, h)=l_{g}^{h},
\end{gathered}
$$

where $l_{g}^{h}$ is defined by $l_{g}^{h}(k, m)=\left(l_{g}(k), l^{h}(m)\right)=(g k, h m)$ for all $(k, m) \in G \times G$. It can easily shown that $l_{g}^{h}$ is a permutation.

We want to show that $\Phi$ is an injective homomorphism.

First, suppose that $\Phi(g, h)=\Phi(k, m)$, then $l_{g}^{h}=l_{k}^{m}$ as functions mapping $G \times G$ to $G \times G$. For functions to be equal they must equal at every point, and hence $l_{g}^{h}(e, e)=l_{k}^{m}(e, e)$ implying $(g, h)=$ $(g e, h e)=(k e, m e)=(k, m)$, and hence $\Phi$ is injective.

It is easily seen that $\Phi$ is a homomorphism just as similar to that one in the equation (1).

Thus, we have an embedding of $G \times G$ into the group of all permutations of the set $G \times G$.

Definition 8. Let $G$ be a group doubly acting on a set $X$ by means of ". " and " 。” and let $x \in X$. Then, the double orbit of $x$ or the double orbit through $x$, written $\operatorname{DOrb}_{G}(x)$, is the set

$$
\operatorname{DOrb}_{G}(x)=\operatorname{Orb}_{(G, \cdot)} x \cap \operatorname{Orb}_{\left(G,{ }^{\circ}\right)} x=\left\{y \in X: g \cdot x=y=h^{\circ} x, g, h \in G\right\} .
$$

Example 6. Let us consider the double action $(G, \cdot, \circ, G)$ in the Example 5. with the symmetric group $G=$ $S_{3}$. Then we have the orbits $\operatorname{Orb}_{\left(S_{3},\right)}(e)=\{e\}, \operatorname{Orb}_{\left(S_{3},\right)}((12))=\operatorname{Orb}_{\left(S_{3},\right)}((13))=\operatorname{Orb}_{\left(S_{3}, \cdot\right)}((23))=$ $\{(12),(13),(23)\}, \quad \operatorname{Orb}_{\left(S_{3},\right)}((123))=\operatorname{Orb}_{\left(S_{3},{ }^{\prime}\right.}((132))=\{(123),(132)\} \quad$ and $\operatorname{Orb}_{\left(S_{3},{ }^{\circ}\right)}(e)=\{e\}$, $\operatorname{Orb}_{\left(S_{3},{ }^{\circ}\right)}((12))=\{(12)\}, \quad \operatorname{Orb}_{\left(S_{3},{ }^{\circ}\right)}((13))=\{(13)\}, \quad \operatorname{Orb}_{\left(S_{3},{ }^{\circ}\right)}((23))=\{(23)\}, \quad \operatorname{Orb}_{\left(S_{3},{ }^{\circ}\right)}((123))=$ $\{(123)\}, \operatorname{Orb}_{\left(S_{3},{ }^{\circ}\right)}((132))=\{(132)\}$. Hence the double orbits of double action $\left(S_{3},, \circ, S_{3}\right)$ are the sets of $\operatorname{DOrb}_{S_{3}}(e)=\{e\}, \operatorname{DOrb}_{S_{3}}((12))=\{(12)\}, \operatorname{DOrb}_{S_{3}}((13))=\{(13)\}, \operatorname{DOrb}_{S_{3}}((23))=\{(23)\}$, $\operatorname{DOrb}_{S_{3}}((123))=\{(123)\}, \operatorname{DOrb}_{S_{3}}((132))=\{(132)\}$.

Example 7. Let us consider the double action $(G, \cdot, \circ, G)$ in the Example 4. with the symmetric group $G=$ $S_{3}$. In this case, with a standard calculation it is easily seen that all double orbits of $S_{3}$ for each $x \in S_{3}$ will be $S_{3}$.

Proposition 4. Let $G$ be a group and $X$ a double $G-$ set. Then, for every $x, y, z \in X$

i) $x$ is in the double orbit through $x$. 
ii) if $y$ is in the double orbit through $x$, then $x$ is in the double orbit through $y$.

iii) if $y$ is in the double orbit through $x$ and $z$ is in the double orbit through $y$, then $z$ is also in the double orbit through $x$.

Proof. i) For any elements $g, h \in G$ and $x \in X$, we have $g \cdot x=x \Rightarrow g=e$ and $h^{\circ} x=x \Rightarrow h=e$. Since $e \in G, x$ is in the double orbit through $x$.

ii) If $y$ is in the double orbit through $x$, then we have $g \cdot x=y$ and $h^{\circ} x=y$ for some $g, h \in G$. Hence

$$
\begin{aligned}
g \cdot x=y & \Rightarrow\left(g^{-1}\right) \cdot(g \cdot x)=\left(g^{-1}\right) \cdot y \\
& \Rightarrow\left(g^{-1} g\right) \cdot x=\left(g^{-1}\right) \cdot y \\
& \Rightarrow e \cdot x=\left(g^{-1}\right) \cdot y \\
& \Rightarrow x=\left(g^{-1}\right) \cdot y
\end{aligned}
$$

and

$$
\begin{aligned}
h^{\circ} x=y & \Rightarrow\left(h^{-1}\right)^{\circ}\left(h^{\circ} x\right)=\left(h^{-1}\right)^{\circ} y \\
& \Rightarrow\left(h^{-1} h\right)^{\circ} x=\left(h^{-1}\right)^{\circ} y \\
& \Rightarrow e^{\circ} x=\left(h^{-1}\right)^{\circ} y \\
& \Rightarrow x=\left(h^{-1}\right)^{\circ} y .
\end{aligned}
$$

From the equations (2) and (3), $x$ is also in the double orbit through $y$.

iii) If $y$ is in the double orbit through $x$, then we have

$$
y=g \cdot x=h^{\circ} x \text { for } \exists g, h \in G,
$$

and $z$ is in the double orbit through $y$, then we have

$$
z=m \cdot y=n^{\circ} y \text { for } \exists m, n \in G .
$$

Hence

$$
z=m \cdot y \Rightarrow z=m \cdot(g \cdot x) \Rightarrow z=(m g) \cdot x
$$

and

$$
z=n^{\circ} y \Rightarrow z=n^{\circ}\left(h^{\circ} x\right) \Rightarrow z=(n h)^{\circ} x .
$$

Since $m g, n h \in G, z$ is in the double orbit through $x$. Hence, the proof is completed.

Thus, we obtain a relation on $X$ defined by 


$$
y \sim x \Leftrightarrow y \text { is in the double orbit through } x .
$$

Corollary 1. The above relation is an equivalence relation.

The equivalence classes under this equivalence relation are called double orbits.

Proposition 5. Let $G$ be a group and $X$ a double $G$ - set. The disjoint double orbits under the above equivalence relation form a partition of $X$.

Proof. From Proposition.4 (i), we have $x \in \operatorname{DOrb}_{G} x$, namely, for $e \in G e \cdot x=x=e^{\circ} x$. So it is obvious that $X \subset \cup_{x \in X} \operatorname{DOrb}_{G} x$. Hence, we suppose that $\operatorname{DOrb}_{G} x \cap D \operatorname{Orb}_{G} y \neq \emptyset$. Then, for at least one element $z \in \operatorname{DOrb}_{G} x \cap D \operatorname{Drb}_{G} y$, there exist $g_{1}, g_{2}, h_{1}, h_{2} \in G$ such that

$$
z=g_{1} \cdot x=h_{1}^{\circ} x \text { and } z=g_{2} \cdot y=h_{2}^{\circ} y \text {. }
$$

We want to show that $D \operatorname{Orb}_{G} x=D \operatorname{Orb}_{G} y$. For this, it suffices to show that $\operatorname{DOrb}_{G} x \subset D \operatorname{Drb}_{G} y$. Thus, we conclude that $D \operatorname{Orb}_{G} x=D \operatorname{Orb}_{G} y$ by symmetry.

From the hypothesis, for any element $u \in \operatorname{DOrb}_{G} x$, there exist $g, h \in G$ such that $u=g \cdot x=h^{\circ} x$. Since $x=\left(g_{1}^{-1}\right) \cdot z=\left(h_{1}^{-1}\right)^{\circ} z$, we have

$$
u=g \cdot x \Rightarrow u=g \cdot\left(\left(g_{1}^{-1}\right) \cdot z\right)=\left(g g_{1}^{-1}\right) \cdot z=\left(g g_{1}^{-1}\right) \cdot\left(g_{2} \cdot y\right)=\left(g g_{1}{ }^{-1} g_{2}\right) \cdot y
$$

and

$$
u=h^{\circ} x \Rightarrow u=h^{\circ}\left(\left(h_{1}^{-1}\right)^{\circ} z\right)=\left(h h_{1}{ }^{-1}\right)^{\circ} z=\left(h h_{1}{ }^{-1}\right)^{\circ}\left(h_{2}^{\circ} y\right)=\left(h h_{1}{ }^{-1} h_{2}\right)^{\circ} y
$$

This means that $u \in \operatorname{DOrb}_{G} y$. Thus, we obtain $\operatorname{DOrb}_{G} x \subset D \operatorname{Drb}_{G} y$.

By symmetry, we obtain $D \operatorname{Orb}_{G} x=D \operatorname{Orb}_{G} y$. Consequently, if the double orbits $D \operatorname{Orb}_{G} x$ and $D O r b_{G} y$ share even one point in common, then they are equal and thus a partition.

Definition 9. Let $X$ be a double $G-$ set. Let $x \in X$. The stabilizer, denoted $G_{x}$, of $x$ is the set

$$
G_{x}=\left\{(g, h) \in G \times G: g \cdot x=h^{\circ} x=x, \quad g, h \in G\right\}
$$

which is the subset of the product group $G \times G$.

Proposition 6. The stabilizer $G_{x}$ of $x$ is the subgroup of the product group $G \times G$.

Proof. $G_{x}$ is nonempty, since $(e, e) \in G_{x}$. Also, if $\left(g_{1}, h_{1}\right),\left(g_{2}, h_{2}\right) \in G_{x}$, then we have

$$
g_{1} \cdot x=h_{1}^{\circ} x=x \text { and } g_{2} \cdot x=h_{2}^{\circ} x=x \text {. }
$$

Hence

$$
g_{1} \cdot x=x \Rightarrow g_{1} \cdot\left(g_{2} \cdot x\right)=x \Rightarrow\left(g_{1} g_{2}\right) \cdot x=x
$$

and

$$
h_{1}^{\circ} x=x \Rightarrow h_{1}^{\circ}\left(h_{2}^{\circ} x\right)=x \Rightarrow\left(h_{1} h_{2}\right)^{\circ} x=x .
$$


Thus, $\left(g_{1} g_{2}, h_{1} h_{2}\right) \in G_{x} \subset G \times G$. It is obvious that the multiplication in $G \times G$ is defined by

$$
\left(g_{1}, h_{1}\right)\left(g_{2}, h_{2}\right)=\left(g_{1} g_{2}, h_{1} h_{2}\right), \quad \text { for all }\left(g_{1}, h_{1}\right),\left(g_{2}, h_{2}\right) \in G \times G .
$$

Thus, $G_{x}$ is closed under the multiplication.

On the other hand, if $\left(g_{1}, h_{1}\right) \in G_{x}$, then we have $g_{1} \cdot x=h_{1}{ }^{\circ} x=x$. Hence,

$$
\begin{aligned}
g_{1} \cdot x=x & \Rightarrow\left(g_{1}^{-1}\right) \cdot\left(g_{1} \cdot x\right)=\left(g_{1}^{-1}\right) \cdot x \\
& \Rightarrow\left(g_{1}^{-1} g_{1}\right) \cdot x=\left(g_{1}^{-1}\right) \cdot x \\
& \Rightarrow e \cdot x=\left(g_{1}^{-1}\right) \cdot x \\
& \Rightarrow x=\left(g_{1}^{-1}\right) \cdot x
\end{aligned}
$$

and

$$
\begin{aligned}
h_{1}^{\circ} x=x & \Rightarrow\left(h_{1}^{-1}\right)^{\circ}\left(h_{1}^{\circ} x\right)=\left(h_{1}^{-1}\right)^{\circ} x \\
& \Rightarrow\left(h_{1}^{-1} h_{1}\right)^{\circ} x=\left({h_{1}}^{-1}\right)^{\circ} x \\
& \Rightarrow e^{\circ} x=\left(h_{1}^{-1}\right)^{\circ} x \\
& \Rightarrow x=\left(h_{1}^{-1}\right)^{\circ} x .
\end{aligned}
$$

So, it follows $\left(g_{1}^{-1}, h_{1}^{-1}\right) \in G_{x}$. Thus, $G_{x}$ is closed under the inversion.

Consequently, the stabilizer $G_{x}$ is the subgroup of $G \times G$.

Example 8. Let us consider the double action $(G, \cdot, \circ, G)$ in the Example 5. with the symmetric group $G=$ $S_{3}$. Then, we obtain the stabilizer groups $\left(S_{3}\right)_{e}=\left\{(e, e\},\left(S_{3}\right)_{(12)}=\{((12),(12))\},\left(S_{3}\right)_{(13)}=\right.$ $\{((13),(13))\},\left(S_{3}\right)_{(23)}=\{((23),(23))\},\left(S_{3}\right)_{(123)}=\{((123),(123))\},\left(S_{3}\right)_{(132)}=\{((132),(132))\}$

Proposition 7. Let $G$ be a group doubly acting on a set $X$ by means of ". " and "”。' Then, for every $\left(g_{1}, h_{1}\right),\left(g_{2}, h_{2}\right) \in G_{x}$

$$
g_{1} \cdot x=g_{2} \cdot x \text { and } h_{1}^{\circ} x=h_{2}^{\circ} x
$$

$$
\begin{gathered}
\Leftrightarrow \\
\left(g_{1}, h_{1}\right) \text { and }\left(g_{2}, h_{2}\right) \text { lie in the same left coset of } G_{x} \text {. }
\end{gathered}
$$

Proof. The conditions $g_{1} \cdot x=g_{2} \cdot x$ and $h_{1}{ }^{\circ} x=h_{2}{ }^{\circ} x$ are equivalent to $x=\left(g_{1}{ }^{-1} g_{2}\right) \cdot x=$ $\left(h_{1}^{-1} h_{2}\right)^{\circ} x$, which means $\left(g_{1}^{-1} g_{2}, h_{1}{ }^{-1} h_{2}\right) \in G_{x}$. Therefore, $\left(g_{1}, h_{1}\right)$ and $\left(g_{2}, h_{2}\right)$ act doubly in the same way on $x$ if and only if $\left(g_{1}, h_{1}\right)$ and $\left(g_{2}, h_{2}\right)$ lie in the same left coset of $G_{x}$.

Definition 10. Double actions of $G$ on sets $X$ and $X^{\prime}$ are called equivalent if there is a bijection $\phi: X \rightarrow X^{\prime}$ such that 


$$
\phi(g \cdot x)=g \cdot(\phi(x)) \text { and } \phi\left(h^{\circ} x\right)=h^{\circ}(\phi(x))
$$

for every $g, h \in G$ and $x \in X$.

Let us now give some types of the double actions.

Definition 11. The double action of a group $G$ on a set $X$ by means of the actions ". " and "。” is called transitive if there is an element $(g, h) \in G \times G$ such that

$$
g \cdot x=y=h^{\circ} x \text { for any } x, y \in X .
$$

Example 9. Let us consider the double action $(G, \cdot, \circ, G)$ in the Example 4. with the symmetric group $G=$ $S_{3}$. By the Example 7., the double action $\left(S_{3}, \circ, \circ, S_{3}\right)$ has unique orbit, so this double action is transitive.

Definition 12. The double action of a group $G$ on a set $X$ by means of the actions ". " and " $\circ$ " is called faithful (or effective) if there is a point $x \in X$ such that

$$
g_{1} \cdot x \neq g_{2} x \text { and } h_{1}^{\circ} x \neq h_{2}^{\circ} x \text { for any }\left(g_{1}, h_{1}\right),\left(g_{2}, h_{2}\right) \in G \times G .
$$

Definition 13. The double action of a group $G$ on a set $X$ by means of the actions ". " and "。” is called free if for $\left(g_{1}, h_{1}\right),\left(g_{2}, h_{2}\right) \in G \times G$ if there exists $x \in X$ such that $g_{1} \cdot x=g_{2} \cdot x$ and $h_{1}{ }^{\circ} x=h_{2}{ }^{\circ} x$ imply $\left(g_{1}, h_{1}\right)=\left(g_{2}, h_{2}\right)$.

Definition 14. The double action of a group $G$ on a set $X$ by means of the actions ". " and " $\circ$ " is called regular if it is both free and transitive. Equivalently, for any $x, y \in X$ there exists a unique $(g, h) \in G \times$ G such that $g \cdot x=y=h^{\circ} x$.

Example 10. The double action in Example 4. is faithful. Indeed, let us consider the actions

$$
\begin{gathered}
\cdot: G \times G \rightarrow G \\
(g, x) \mapsto g x=g x,
\end{gathered}
$$

and

$$
\begin{gathered}
\circ: G \times G \rightarrow G \\
(h, x) \mapsto h^{\circ} x=x h^{-1} .
\end{gathered}
$$

There is a need to show that there exists an $x \in X$ such that

$$
g_{1} \cdot x \neq g_{2} \cdot x \text { and } h_{1}^{\circ} x \neq h_{2}^{\circ} x
$$

when $\left(g_{1}, h_{1}\right) \neq\left(g_{2}, h_{2}\right)$.

Let $g_{1} \neq g_{2}$. Then

$$
\left.\begin{array}{c}
g_{1} \cdot x=g_{1} x \\
g_{2} \cdot x=g_{2} x
\end{array}\right\} \Rightarrow g_{1} x \stackrel{?}{=} g_{2} x \Rightarrow g_{1} x x^{-1} \stackrel{?}{=} g_{2} x x^{-1} \Rightarrow g_{1} e \stackrel{?}{=} g_{2} e \Rightarrow g_{1} \neq g_{2}
$$

and 


$$
\left.\begin{array}{c}
h_{1}^{\circ} x=x h_{1}^{-1} \\
h_{2}^{\circ} x=x h_{2}^{-1}
\end{array}\right\} \Rightarrow x h_{1}^{-1} \stackrel{?}{=} x h_{2}^{-1} \Rightarrow x^{-1} x h_{1}^{-1} \stackrel{?}{=} x^{-1} x h_{2}^{-1} \Rightarrow e h_{1}^{-1} \stackrel{?}{=} e h_{2}^{-1} \Rightarrow h_{1}^{-1} \stackrel{?}{=} h_{2}^{-1} \Rightarrow h_{1} \neq h_{2}
$$

Therefore, the double action is faithful.

Example 11. Let $G$ be a group and $H \subset G$ a subgroup. Let $G / H=\{x H: x \in G\}$ be set of the left cosets. There is a natural action of $G$ on $G / H$ with the left multiplication:

$$
\begin{gathered}
\cdot: G \times G / H \rightarrow G / H \\
(g, x H) \mapsto g \cdot(x H)=(g x) H .
\end{gathered}
$$

Let us define another action as trivial action:

$$
\begin{gathered}
\circ: G \times G / H \rightarrow G / H \\
(k, x H) \mapsto k^{\circ}(x H)=x H .
\end{gathered}
$$

Then, we have

$$
g \cdot\left(k^{\circ}(x H)\right)=g \cdot(x H)=(g x) H=k^{\circ}((g x) H)=k^{\circ}(g \cdot(x H)) .
$$

Thus, $G$ acts doubly on $G / H$ by means of the actions " . " and " 。 ".

\section{REFERENCES}

[1] Alp M., Pullback Crossed Modules of Algebroids, Iran. J. Sci. Technol. Trans. A Sci., No.A1, 32 (2008) 1-5.

[2] Brown R., Topology and Groupoids, BookSurge LLC, Deganwy, United Kingdom, (2006).

[3] Derek J.S.R., A Course in the Theory of Groups, Springer-Verlag, New York, USA, (1996).

[4] Gürsoy M.H., Actions of Vector Groupoids, Bull. Iranian Math. Soc., 40-3 (2014) 565-582.

[5] Norrie K., Actions and Automorphisms of Crossed Modules, Bull. Soc. Math. France, 118 (1990) 129146. 